\title{
Drivers and barriers of water intake in preschool children in a one-year longitudinal field study in Poland
}

\author{
Jeanne H. Bottin ${ }^{1}$, Sabine Boesen-Mariani ${ }^{2}$, Isabelle Guelinckx ${ }^{1}$, Bradley Franks ${ }^{3}$, \\ Quentin Dornic ${ }^{4}$, Aukje Verhoeven ${ }^{5}$ and Saadi Lahlou ${ }^{3}$ \\ ${ }^{1}$ Hydration and Health Department, Danone Research, Palaiseau, France, \\ ${ }^{2}$ Sensory and Behavior Science Department, Nutricia Research, Utrecht, Netherlands, \\ ${ }^{3}$ Department of Psychological and Behavioural Science, London School of Economics and Political Science, London, \\ United Kingdom, \\ ${ }^{4}$ Biometrics and Data Sciences, Danone Research, Palaiseau, France and \\ ${ }^{5}$ Sensory and Behavior Science Department, Danone Research, Palaiseau, France
}

\begin{abstract}
Background: Many children consume too little water and too many sugar-sweetened beverages (SSBs), with potential negative consequences for health. Sustainably increasing water intake is therefore relevant, however challenging.

Objective: Explore drivers of and barriers to water intake during a one-year field study aiming to increase plain water consumption using Installation Theory in Polish preschool children.

Method: Caregivers of children (3-6 yrs) completed questionnaires on the child's preference for water and SSBs, water drinking habit strength (Self-Report Habit Index, range 1 (low)-5 (high habit)), and barriers to drinking water, at baseline and after one year. Children were first allocated to one of three interventions: CONTROL: no intervention; INFO: online coaching sessions on water health benefits ( 3 weeks); INFO + W: similar coaching sessions and home water delivery ( 3 weeks). After 3 months, half of INFO and INFO + W subjects were exposed to an online discussion forum ( 3 weeks) (+ SOCIAL); the other half received no further intervention (-SOCIAL).
\end{abstract}

Results: 334 children (age: $4.4 \pm 1.2$ yrs; $50 \%$ female) completed the study. At baseline, $72 \%$ attended kindergarten, among which $48 \%$ had access to water during the day. Drinking water during class was not allowed for $41 \%$. The proportion of children who liked or loved water increased from $28 \%$ at baseline to $67 \%$ after one year. Conversely, that of children who liked or loved SSBs decreased from $58 \%$ at baseline to $45 \%$ after one year. The increase in preference for water was greater in groups who initially received INFO + W compared to CONTROL $(\mathrm{p}=0.004)$. The mean drinking water habit score increased from 2.3 at baseline to 3.0 across all groups with a larger increase in INFO + W + SOCIAL $(+28 \%)$ compared to CONTROL $(+16 \%)(p=0.001)$. At baseline, the strongest barriers to drinking water were (1) Drinking large amounts of other beverages (72\%), (2) A preference for other beverages such as SSBs $(71 \%)$, (3) Not being aware that they should drink more water $(61 \%)$, and (4) Not being used to drinking water or water having no taste (both 55\%). Most barriers strongly decreased over the course of the study with no specific effect of the interventions.

Conclusion: An intervention aiming to increase water in pre-school children was able to modulate the preference for water, habit to drink water, and barriers to drinking water in preschool children with unhealthy drinking habits. Providing information and facilitating access to water were the most important contributors to improving healthy hydration habits.

\section{Conflict of Interest}

JHB, QD, IG, AV are full-time employees of Danone Research and SBM of Nutricia Research. 